Coherent X-ray radiation excited by a beam of relativistic electrons in a layered periodic structure

To cite this article: S. Blazhevich et al 2020 JINST 15 C05075

View the article online for updates and enhancements.

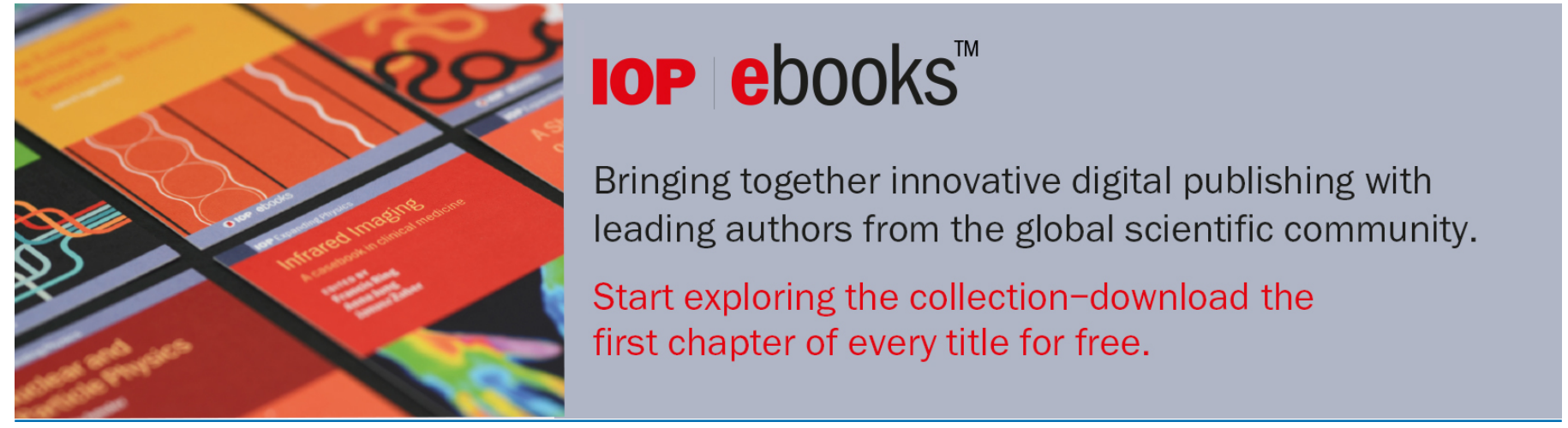

This content was downloaded from IP address 188.170 .217 .49 on 02/09/2020 at 07:39 


\title{
Coherent X-ray radiation excited by a beam of relativistic electrons in a layered periodic structure
}

\author{
S. Blazhevich, ${ }^{a}$ A. Noskov ${ }^{a, b, 1}$ and O. Shevchuk ${ }^{a}$ \\ ${ }^{a}$ Belgorod State University, Pobedy Str., 85, Belgorod 308015, Russia \\ ${ }^{b}$ Belgorod State Technological University named after V.G. Shukhov, \\ Kostyukova Str., 46, Belgorod 308012, Russia \\ E-mail: noskovbupk@mail.com
}

Abstract: The dynamic theory of coherent X-ray radiation, excited in a periodic layered medium by a divergent beam of relativistic electrons in the Bragg scattering geometry, is developed. In framework of the two-wave approximation of the dynamic theory of diffraction, expressions describing the spectral-angular and angular distributions of parametric X-ray radiation (PXR) and diffracted transition radiation (DTR) are obtained taking into account the multiple scattering of the relativistic electrons by the atoms of the target. Based on the expressions obtained, the possibilities of the manifestation of the effects of dynamical diffraction in coherent X-rays were investigated. The influence of the asymmetry of the electron Coulomb field reflection with respect to the target surface (the reflecting layers situate nonparallel to target surface) on the spectral-angular characteristics of the PXR and the DTR under conditions of the electron multiple scattering is estimated. It is shown that at a fixed Bragg angle the width of the PXR spectrum increases when the angle of incidence of the electron on the target decreases, which leads to an increase in the PXR angular density. In the same conditions the width of the frequency domain of total external reflection and the amplitude of the DTR spectrum also increase, which leads to a significant increase in the angular density of the DTR. The obtained analytical expressions can be used to determine the optimal parameters of the experiment on confirmation of the predicted dynamic effects.

KeYwords: Models and simulations; Radiation calculations

\footnotetext{
${ }^{1}$ Corresponding author.
} 


\section{Contents}

1 Introduction 1

2 Geometry of the radiation process 2

3 Spectral-angular density 3

4 The influence of multiple scattering of relativistic electrons 4

5 Conclusions $\quad 8$

\section{Introduction}

The coherent X-ray radiation excited by relativistic electrons in structured media is of current interest for physicists and engineers from points of view both its fundamental research and its different applications.

Parametric X-ray radiation (PXR) in a periodic layered medium arises due to diffraction of pseudo-photons of the Coulomb field of a relativistic electron by the target layers, similar to how PXR occurs in a single-crystal due to the diffraction by a system of parallel atomic planes $[1,2]$.

Diffracted transition radiation (DTR) is the result of diffraction of transition radiation photons generated near the entrance surface of the target on the target layers by analogy with the DTR in a single crystal $[3,4]$.

The dynamic theory of radiation of relativistic electrons in periodic layered media in the case of symmetric reflection [5] well describes the experimental data presented in [6]. In the case of symmetric reflection, the layers are parallel to the target surface. The process of coherent Xray radiation by a single relativistic electron in a periodic layered medium for the general case of asymmetric reflection of the electron field relative to the target surface in the Laue scattering geometry was considered in [7], and in the Bragg scattering geometry in [8]. In the work [7] it was shown that the yield of the coherent radiation excited in layered target is considerably bigger than in single-crystal one in the analogous conditions. This result opens the new perspectives for application of such target in alternative source of X-ray radiation.

In [9], the dynamic theory of coherent X-ray radiation excited when a diverging beam of relativistic electrons passes through a periodic layered target was developed. For the Bragg scattering geometry, expressions are obtained that describe the spectral-angular characteristics of coherent Xray radiation.

In the present work, we have considered coherent X-ray radiation from a beam of the relativistic electrons crossing the target in the form of a periodic layered medium in the Bragg scattering geometry. The consideration has made for a general case of not parallel situation of the medium layers in respect to the target surface. The main question under consideration in this work is how the multiple scattering of the relativistic electrons affects the dynamical effects in the spectral-angular distribution of the coherent radiation excited in the layered target. With this goal we investigate 
the effect of the relativistic electrons multiple scattering in the target by the medium atoms on the spectral-angular characteristics of PXR and DTR. The significant growth of the coherent X-ray radiation yield from the target with periodic layered structure in comparison with monocrystalline one opens a perspective of application of such a target in intensive X-ray sources based on the interaction of relativistic electron beams with periodic structures. The results of the investigation of the radiation process features in such structures will be very useful in constructing the alternative $\mathrm{X}$-ray source based on interaction of relativistic electrons with periodic layered media.

\section{Geometry of the radiation process}

Let us consider a beam of relativistic electrons with divergence $\psi_{0}$ intersecting a layered target in a Bragg scattering geometry (figure 1). We consider the transverse size of the electron beam incident on the target as very small and do not take into account its effect on the radiation angular distribution. Periodic layered medium consists of alternating layers with thicknesses $l_{1}$ and $l_{2}$ with dielectric susceptibilities, respectively $\chi_{1}$ and $\chi_{2}\left(T=l_{1}+l_{2}\right.$ is the period of the layered target). The reflecting layers are located at a certain angle $\delta$ to the target surface (figure 1), which corresponds to the case of asymmetric reflection of the radiation field $(\delta=0$ is a special case of symmetric reflection). We consider that the process of the coherent radiation excited in the target by each electron in the beam is independent. Therefore, the spectral-angular density of radiation, generated by the electron beam can be obtained by averaging the expression for the spectral-angular density of the radiation generated by a separate electron in the beam through all its possible trajectories in the target. As well as the angular distribution of electron in the beam and radiation is very narrow we introduce the vector variables $\boldsymbol{\psi}, \boldsymbol{\theta}$ and $\boldsymbol{\theta}_{0}$ in accordance with the definitions of the velocity of the relativistic electron $\mathbf{V}$ and unit vectors: $\mathbf{n}$ - in the direction of the photon momentum emitted near the direction of the electron velocity vector, and $\mathbf{n}_{\mathbf{g}}$ - in the direction of Bragg scattering, using the small-angle approximation:

$$
\begin{aligned}
& \mathbf{V}=\left(1-\frac{1}{2} \gamma^{-2}-\frac{1}{2} \psi^{2}\right) \mathbf{e}_{1}+\psi, \quad \mathbf{e}_{1} \psi=0, \quad \mathbf{n}=\left(1-\frac{1}{2} \theta_{0}^{2}\right) \mathbf{e}_{1}+\boldsymbol{\theta}_{\mathbf{0}}, \\
& \mathbf{e}_{1} \boldsymbol{\theta}_{0}=0 \\
& \mathbf{e}_{1} \mathbf{e}_{2}=\cos 2 \theta_{B}, \quad \mathbf{n}_{\mathbf{g}}=\left(1-\frac{1}{2} \theta^{2}\right) \mathbf{e}_{2}+\boldsymbol{\theta}, \quad \mathbf{e}_{2} \boldsymbol{\theta}=0
\end{aligned}
$$

where $\theta \approx|\boldsymbol{\theta}|$ is the radiation angle counted from the axis $\mathbf{e}_{2}$ of the radiation detector, $\psi \approx|\psi|$ is the deviation angle of the rectilinear trajectory of the electron from the beam axis $\mathbf{e}_{1}, \theta_{0} \approx\left|\boldsymbol{\theta}_{\mathbf{0}}\right|$ is the angle between the direction $\mathbf{n}$ of the incident pseudo photon propagation and the axis $\mathbf{e}_{1}$, $\gamma=1 / \sqrt{1-V^{2}} \equiv E_{e} / m$ is the Lorentz factor of the relativistic electron. In accordance with the $\mathbf{V}$ definition in (2.1), the $|\mathbf{V}|=V$ for any value of the angle $\psi$. The $\mathbf{e}_{2}$ axis determines the direction of specular reflection of photons incident on the target along the axis $\mathbf{e}_{1}$. In our notation the Bragg angle $\theta_{B}$ is the angle between the electron beam axis $\mathbf{e}_{1}$ and the reflecting layers in the target (see in figure 1). So the expression $\mathbf{e}_{1} \mathbf{e}_{2}=\cos 2 \theta_{B}$ in (2.1) is the definition of $\theta_{B}$ as well. The corresponding photon energy is defined as $\omega_{B}=\frac{g}{2 \sin \theta_{B}}$. The introduced vectors are considered as the sum of components parallel and perpendicular to the plane of the figure: $\boldsymbol{\theta}=\boldsymbol{\theta}_{\|}+\boldsymbol{\theta}_{\perp}$, $\boldsymbol{\theta}_{0}=\boldsymbol{\theta}_{0 \|}+\boldsymbol{\theta}_{0 \perp}, \psi=\psi_{\|}+\psi_{\perp}$. 


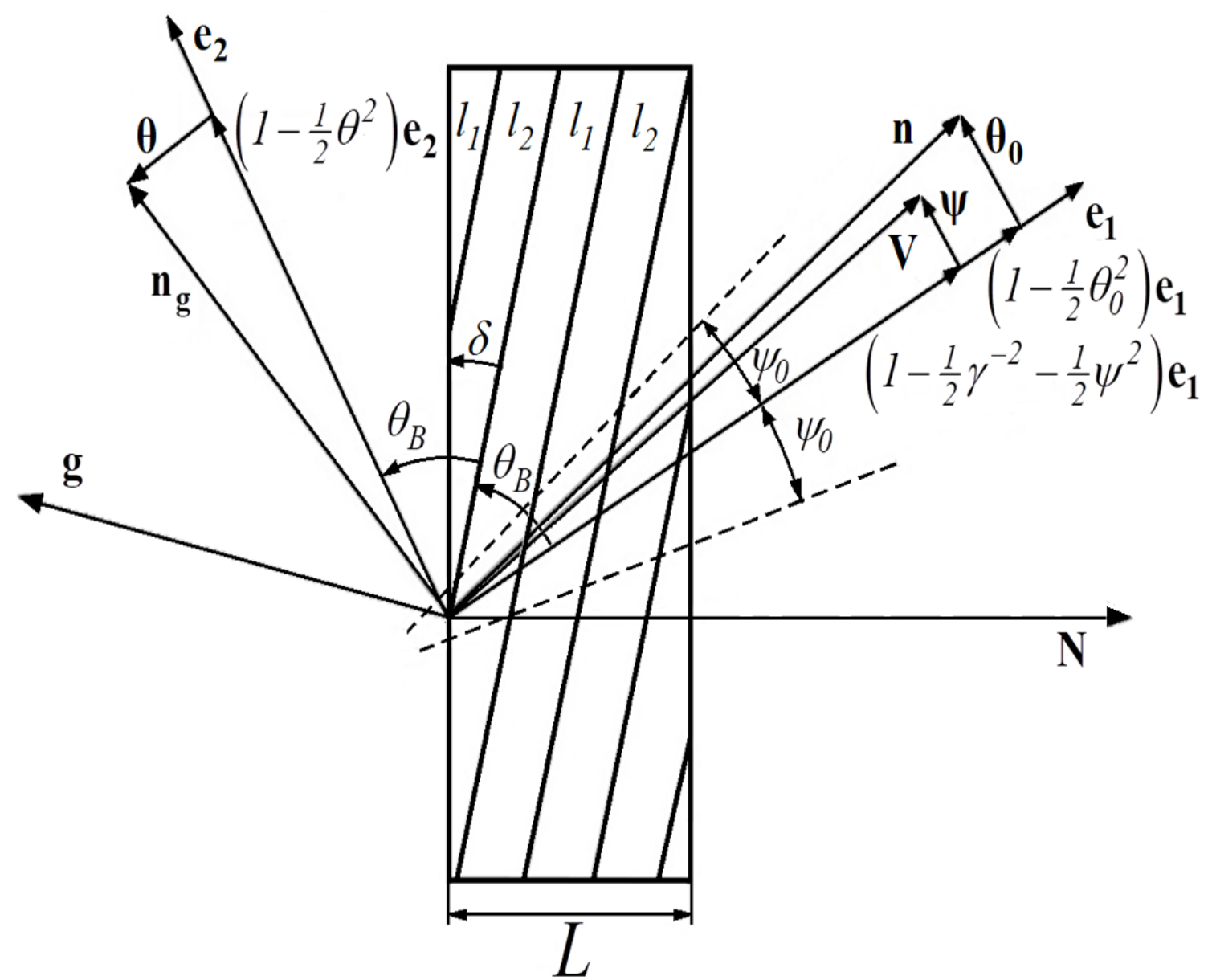

Figure 1. Geometry of the radiation process. $\mathbf{N}$ is normal to the target boundary, $\mathbf{e}_{1}$ is unit vector of the electron beam axis, $\mathbf{e}_{2}$ is unit vector of the Bragg scattering direction, near which PXR and DTR are observed, $\mathrm{L}$ is the target thickness, $\psi_{0}$ is parameter of the electron beam divergence, $\mathbf{n}$ is vector in the direction of diffracted pseudo photon of the relativistic electron coulomb field, $\mathbf{n}_{\mathbf{g}}$ is vector in the direction of diffracted pseudo photon. $\theta_{B}$ is the Bragg angle of the electron beam (angle of mirror reflection of the photon on the beam axis $\mathbf{e}_{1}$ from layers of the target to direction of axis $\mathbf{e}_{2}$ ).

\section{Spectral-angular density}

In the framework of the two-wave approximation of the dynamic theory of diffraction, the expressions that describe the spectral-angular density of PXR and DTR in the case of a thin non-absorbing target were obtained in [9]:

$$
\begin{aligned}
& \omega \frac{d^{2} N_{\mathrm{PXR}}^{(s)}}{d \omega d \Omega}=\frac{e^{2}}{\pi^{2}} \frac{\Omega^{(s) 2}}{\left(\Omega-\chi_{0}^{\prime}\right)^{2}} \frac{\left(\xi^{(s)}+\sqrt{\xi^{(s) 2}-\varepsilon}\right)^{2}}{\xi^{(s) 2}-\varepsilon+\varepsilon \sin ^{2}\left(\frac{b^{(s)} \sqrt{\xi^{(s) 2}-\varepsilon}}{\varepsilon}\right)} \frac{\sin ^{2}\left(\frac{b^{(s)}}{2}\left(\frac{\xi^{(s)}+\sqrt{\xi^{(s) 2}-\varepsilon}}{\varepsilon}-\sigma^{(s)}\right)\right)}{\left(\frac{\xi^{(s)}+\sqrt{\xi^{(s) 2}-\varepsilon}}{\varepsilon}-\sigma^{(s)}\right)^{2}}, \\
& \omega \frac{d^{2} N_{\mathrm{DTR}}^{(s)}}{d \omega d \Omega}=\frac{e^{2}}{\pi^{2}} \Omega^{(s) 2}\left(\frac{1}{\Omega}-\frac{1}{\Omega-\chi_{0}^{\prime}}\right)^{2} \frac{\varepsilon^{2}}{\xi^{(s)^{2}}-\left(\xi^{(s)^{2}}-\varepsilon\right) \operatorname{coth}^{2}\left(\frac{b^{(s)} \sqrt{\varepsilon-\xi^{(s)^{2}}}}{\varepsilon}\right)}
\end{aligned}
$$


The following notation is introduced in formulas (3.1):

$$
\begin{aligned}
\Omega^{(1)} & =\theta_{\perp}-\psi_{\perp}, \quad \Omega^{(2)}=\theta_{\|}+\psi_{\|}, \quad \Omega=\gamma^{-2}+\left(\theta_{\perp}-\psi_{\perp}\right)^{2}+\left(\theta_{\|}+\psi_{\|}\right)^{2}, \quad b^{(s)}=\frac{1}{2 \sin \left(\theta_{\mathrm{B}}+\delta\right)} \frac{L}{L_{\mathrm{ext}}^{(s)}}, \\
\sigma^{(s)} & =\frac{\pi n}{C^{(s)}\left|\chi_{2}^{\prime}-\chi_{1}^{\prime}\right|\left|\sin \left(\frac{\pi n}{1+r}\right)\right|}\left(\gamma^{-2}+\left(\theta_{\perp}-\psi_{\perp}\right)^{2}+\left(\theta_{\|}+\psi_{\|}\right)^{2}-\chi_{0}^{\prime}\right), \\
\xi^{(s)}(\omega) & =\eta^{(s)}(\omega)+\frac{1+\varepsilon}{2 v^{(s)}}, \quad \eta^{(s)}(\omega)=\frac{2 \pi^{2} n^{2}}{T^{2} \omega_{\mathrm{B}}} L_{\mathrm{ext}}^{(s)}\left(1-\frac{\omega}{\omega_{\mathrm{B}}}\left(1-\theta_{\|} \sqrt{\frac{T^{2} \omega_{\mathrm{B}}^{2}}{\pi^{2} n^{2}}-1}\right)\right), \quad T=l_{1}+l_{2} \\
\varepsilon & =\frac{\sin \left(\theta_{\mathrm{B}}-\delta\right)}{\sin \left(\theta_{\mathrm{B}}+\delta\right)}, \quad L_{\mathrm{ext}}^{(s)}=\frac{1}{\omega} \frac{\pi n}{\left|\sin \left(\frac{\pi n}{1+r}\right)\right|\left|\chi_{2}^{\prime}-\chi_{1}^{\prime}\right| C^{(s)}}, \quad r=\frac{l_{2}}{l_{1}}, C^{(1)}=1, \quad C^{(2)}=\left|\cos 2 \theta_{\mathrm{B}}\right|,
\end{aligned}
$$

where the parameter $b^{(s)}$ is equal to half the electron path in the target $L_{e}=L / \sin \left(\theta_{\mathrm{B}}+\delta\right)$, expressed in the extinction lengths $L_{\text {ext }}^{(s)}$ of X-ray waves in a periodic layered medium. The $\eta^{(s)}(\omega)$ is a linear function of frequency $\omega$. For a fixed value of $\theta_{\mathrm{B}}$, the asymmetry parameter $\varepsilon$ determines the orientation of the input surface of the target relative to the reflecting layers. Index $s=1$ denotes a sigma -polarized radiation wave, and $s=2$ denotes a pi-polarized one. The $n$ is the number of the harmonic of the oscillations in the wave reflected by layered medium, which is presented in the formula $g=\frac{2 \pi}{T} n$, where $g$ is an analog of the length of a reciprocal lattice vector in a crystal, $n=0, \pm 1, \pm 2, \ldots$. The vector $\mathbf{g}$ is perpendicular to the target layers.

We find the angular density of the PXR by integrating expression (3.1a) over the frequency $\omega$, using the relation $\frac{d \omega}{\omega}=-\frac{T^{2} \omega_{\mathrm{B}}}{2 L_{\mathrm{ext}} \pi^{2} n^{2}} d \xi^{(s)}$ that follows from the expression for $\xi^{(s)}(\omega)$ in (3.2).

Since the PXR spectrum is very narrow under the condition $b^{(s)} \gg 1$ (thick target), i.e. when the electrons passes through a large number of diffracting layers in the target, we use the well-known approximation for integration $\frac{\sin ^{2}(a x)}{x^{2}} \rightarrow \pi a \delta(x)$ and obtain the following expression for the PXR angular density

$$
\frac{d N_{\mathrm{PXR}}^{(s)}}{d \Omega}=\frac{e^{2} \omega_{\mathrm{B}}^{3} T^{2} L_{\mathrm{ext}}^{(s)}}{2 \pi^{3} n^{2}} \Omega^{(s) 2} \varepsilon^{2} \frac{\sigma^{(s) 2} \varepsilon-1}{\left(\sigma^{(s) 2} \varepsilon-1\right)^{2}+2 \varepsilon \sigma^{(s) 2}} b^{(s)} .
$$

By a similar way we obtain the expressions that describe the angular density of the DTR

$$
\frac{d N_{\mathrm{DTR}}^{(s)}}{d \Omega}=\frac{e^{2} \omega_{\mathrm{B}}^{3} T^{2} L_{\mathrm{ext}}^{(s)}}{2 \pi^{3} n^{2}} \frac{\Omega^{(s) 2}}{\sigma^{(s) 2}\left(v^{(s)} \sigma^{(s)}-1\right)^{2}} \varepsilon \sqrt{\varepsilon} \tanh \left(\frac{b^{(s)}}{\sqrt{\varepsilon}}\right) .
$$

\section{The influence of multiple scattering of relativistic electrons}

To account for multiple scattering of emitting electrons in a medium, we average the spectral-angular and angular densities of the radiation over the angular distribution of electrons in the beam in the form of a Gaussian function that varies with length of the path through the target due to multiple electron scattering:

$$
f(\psi, t)=\frac{1}{\pi\left(\psi_{0}^{2}+\psi_{s}^{2} t\right)} \cdot e^{-\frac{\psi^{2}}{\psi_{0}^{2}+\psi_{s}^{2} t}}
$$


that is, averaged over an expanding beam of rectilinear trajectories of radiating electrons along the length of the electron path in the target $L_{e} . \psi_{0}$ is the initial divergence of the electron beam. The average square of the multiple electron scattering angle per unit length has the form

$$
\psi_{s}^{2}=\frac{l_{1} \psi_{(1)}^{2}+l_{2} \psi_{(2)}^{2}}{l_{1}+l_{2}},
$$

where $\psi_{(1)}^{2}=\frac{E_{s}^{2}}{m^{2} \gamma^{2}} \frac{1}{L_{R}^{(1)}}\left(1+0.038 \operatorname{Ln}\left(\frac{t}{L_{R}^{(1)}}\right)\right)^{2}, \psi_{(2)}^{2}=\frac{E_{s}^{2}}{m^{2} \gamma^{2}} \frac{1}{L_{R}^{(2)}}\left(1+0.038 \operatorname{Ln}\left(\frac{t}{L_{R}^{(2)}}\right)\right)^{2}$ are the mean squares of multiple scattering angles per unit length in the media under consideration, taking into account their dependence on the path length $t[10]$ in a layered medium, $E_{S} \approx \sqrt{\frac{4 \pi m^{2}}{e^{2}}} \approx 21 \mathrm{MeV}$, $L_{R}^{(1)}$ and $L_{R}^{(2)}$ are the electron radiation lengths in the materials of layer $l_{1}$ and $l_{2}$ correspondently.

The spectral-angular and angular radiation densities are averaged over an expanding beam of rectilinear trajectories of the emitting electron (over the variables $\psi_{\perp}, \psi_{\|}$and $t$ ) according to formulas (3.1a)-(3.1b) and (3.3)-(3.4) respectively:

$$
\begin{aligned}
\left\langle\omega \frac{d^{2} N_{\mathrm{PXR}, \mathrm{DTR},}^{(s)}}{d \omega d \Omega}\right\rangle & =\frac{1}{\pi L_{e}} \int_{0}^{L_{e}} d t \iint d \psi_{\perp} d \psi_{\|} \frac{e^{-\frac{\psi^{2}}{\psi_{0}^{2}+\psi_{s}^{2} t}}}{\psi_{0}^{2}+\psi_{s}^{2} t} \omega \frac{d^{2} N_{\mathrm{PXR}, \mathrm{DTR}}^{(s)}}{d \omega d \Omega} \\
\left\langle\frac{d N_{\mathrm{PXR}, \mathrm{DTR}}^{(s)}}{d \Omega}\right\rangle & =\frac{1}{\pi L_{e}} \int_{0}^{L_{e}} d t \iint d \psi_{\perp} d \psi_{\|} \frac{e^{-\frac{\psi^{2}}{\psi_{0}^{2}+\psi_{s}^{2} t}} \omega \frac{d N_{\mathrm{PXR}, \mathrm{DTR}}^{(s)}}{\psi_{0}^{2}+\psi_{s}^{2} t} .}{d \Omega} .
\end{aligned}
$$

We suppose that the length of the formation of coherent radiation $L_{\mathrm{coh}}=2 \pi V / \omega\left(\gamma^{-2}+\theta^{2}+\frac{\omega_{p}^{2}}{\omega^{2}}\right)$ is equal or longer than the path of the relativistic electron in the target, where $\omega_{p}=\sqrt{\frac{4 \pi n_{e} e^{2}}{m_{e}}}$ is the frequency of electron plasma in the target material; therefore, changes in the angular distribution of incident electrons due to multiple scattering by the atoms of the target will affect the spectralangular characteristics of the DTR similar to how it affects the PXR characteristics. Based on the expressions obtained, we carried out numerical calculations of the spectral-angular and angular densities of the radiation generated in a periodic layered medium consisting of layers of carbon $(\mathrm{C})$ and silicon $(\mathrm{Si})$ by a beam of relativistic electrons with relativistic factor $\gamma=500$. For the angle between the axis of the beam of relativistic electrons and the reflective layers (Bragg angle) the value $\theta_{\mathrm{B}}=2.25^{\circ}$ was chosen corresponding to the Bragg frequency $\omega_{\mathrm{B}}=8 \mathrm{keV}$. We choose the same thickness for both the carbon and silicon layers $l_{1}=l_{2}=10^{-3} \mu \mathrm{m}$. In the calculations, the path of the electron in the target always was considered of the same length $L_{e}=100 \mu \mathrm{m}$ and therefore for different values of asymmetry parameter $\varepsilon$ the thickness of the target was different. In the case of symmetric reflection, when $\varepsilon=1(\delta=0)$ as the thickness of the target was the value $L=3.9 \mu \mathrm{m}$, in the case of asymmetric reflection under chosen $\varepsilon=3(\delta=-0.02)$ for the thickness of the target was used the value $L=1.9 \mu \mathrm{m}$. The calculations were carried out for $\sigma$-polarization $(s=1)$. The used values of the radiation length for carbon (C) and silicon ( $\mathrm{Si})$ are $L_{R}^{(1)}=14.7 \mathrm{~cm}$ and $L_{R}^{(2)}=10.2 \mathrm{~cm}$ correspondently.

The figure 2 shows the curves constructed according to formula (3.1a) describing the spectralangular density of the PXR of a relativistic electron at a fixed observation angle $\boldsymbol{\theta}$, with coordinates 
$\theta_{\perp}=4.7 \mathrm{mrad}, \theta_{\|}=0$, at the maximum of the angular density of the $\operatorname{PXR} \theta_{\perp}=\sqrt{\gamma^{-2}-\chi_{0}^{\prime}}$. The electron velocity was directed along the beam axis $\mathbf{e}_{1}\left(\psi_{\perp}=\psi_{\|}=0\right)$. From figure 2, it follows that in the case of asymmetric reflection at $\delta=-0.02$, the width of the PXR peak is substantially larger than in the case of symmetric reflection at $\delta=0$.

Because the electron path in both cases is the same $L_{e}=100 \mu \mathrm{m}$ and the absorption of emitted photons is absent we conclude that this effect is since the resonance condition determining the PXR peak $\frac{\xi^{(s)}(\omega)+\sqrt{\xi^{(s)}(\omega)^{2}-\varepsilon}}{\varepsilon}-\sigma^{(s)} \approx 0$ (see (3.1a)) changes more slowly with increasing frequency $\omega$ when the asymmetry parameter $\varepsilon$ increases. It should be noted that as the parameter $\varepsilon$ increases at a fixed angle $\theta_{\mathrm{B}}$, the angle between the axis $\mathbf{e}_{1}$ of the electron beam and the target surface decreases.

Figure 3 shows curves describing the spectral-angular densities of PXR for various asymmetries, taking into account multiple scattering of beam electrons by target atoms, with the initial beam divergence in the calculations $\psi_{0}=0.1 \mathrm{mrad}$.

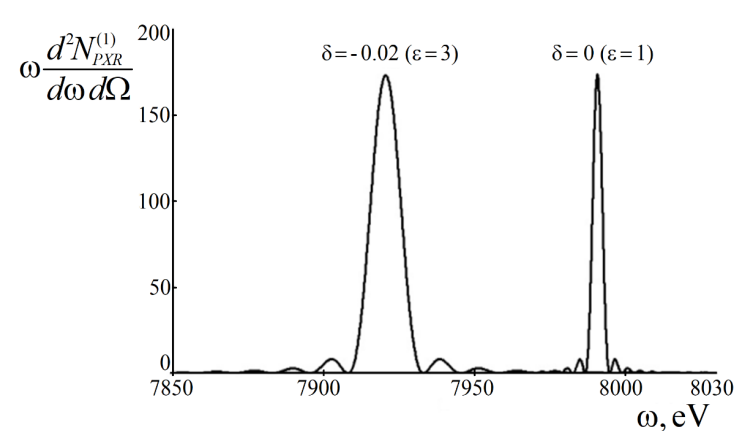

Figure 2. Spectral-angular density of PXR for symmetric and asymmetric cases of reflection for fixes observation angle $\boldsymbol{\theta}, \theta_{\perp}=\sqrt{\gamma^{-2}-\chi_{0}^{\prime}} \approx 4.7 \mathrm{mrad}$, $\theta_{\|}=0$.

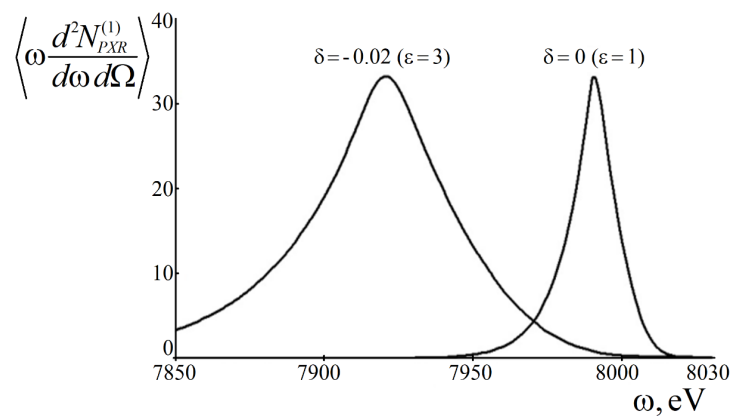

Figure 3. The same as in figure 2 but with consideration of electron multiple scattering. The initial divergence of the relativistic electron beam is $\psi_{0}=0.1 \mathrm{mrad}$.

From a comparison of figure 2 and figure 3 it follows that multiple electron scattering in a periodic layered medium leads to a significant increase in the width of the PXR spectrum and a decrease in its amplitude. But the dynamic effect of broadening of the PXR spectrum due to an increase in the parameter $\varepsilon$ remains in this case as well.

In figure 4 and figure 5, the curves constructed by formulas (3.3) and (4.3b) correspondently present the angular densities of the PXR generated by an electron moving rectilinearly along the axis of the beam $\mathbf{e}_{1}$ (figure 4 ) and by a divergent electron beam in the condition of multiply scattering of the electrons by target atoms (figure 5).

From a comparison of the figures, it follows that multiple scattering under given conditions will affect the angular density of PXR at small angles of observation. This is due to the small rootmean-square angle of multiple scattering of relativistic electrons in the target under consideration. In figure 5 on can see that the effect of the reflection asymmetry on the angular density of PXR remains significant even under conditions of the electron multiple scattering.

Figure 6 shows the curves constructed according to formula (3.1b) describing the spectralangular density of the DTR of a relativistic electron at a fixed angle of observation $\boldsymbol{\theta}$ with coordinates $\theta_{\perp}=2 \mathrm{mrad}, \theta_{\|}=0$. The curves are plotted at the maximum angular density of the DTR 


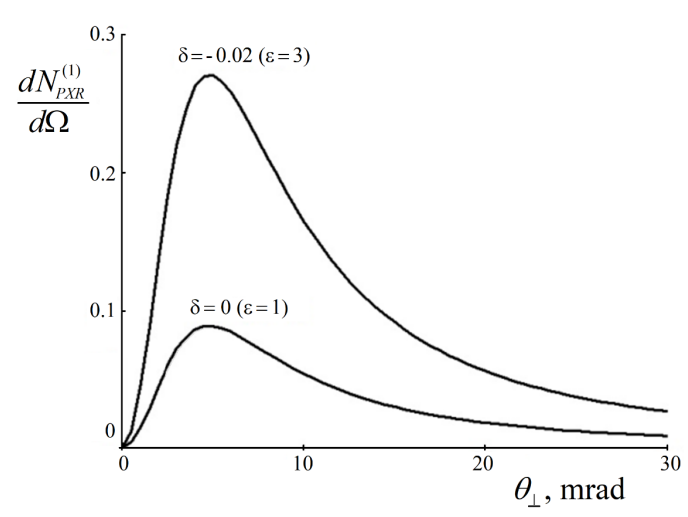

Figure 4. Angular density of PXR for symmetric $(\delta=0, \varepsilon=1)$ and asymmetric $(\delta=-0.02, \varepsilon=3)$ reflection.

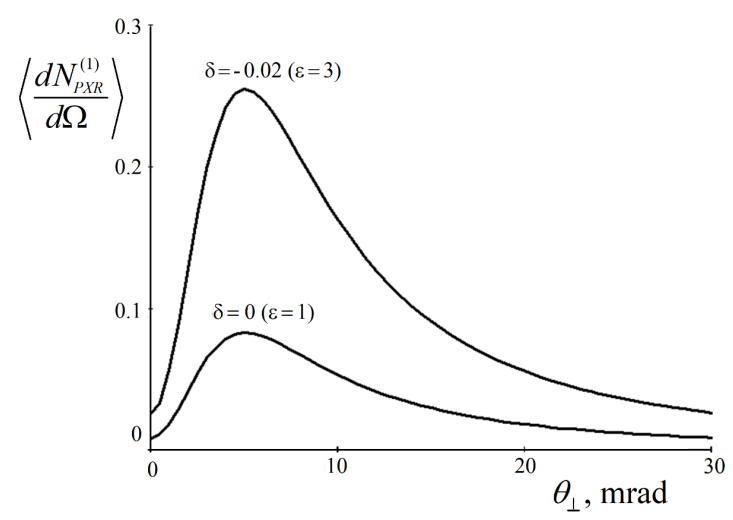

Figure 5. The same as in figure 4 with considering the effect of the electron multiple scattering.

$\psi_{\perp}=\psi_{\|}=0$. From figure 6, it follows that in the case of asymmetric reflection at $\delta=-0.02$ the width and amplitude of the DTR spectrum are significantly larger than in the case of symmetric reflection $(\delta=0)$.

Figure 7 shows curves describing the spectral-angular densities of the DTR considering the multiple scattering of the beam electrons by target atoms, with the initial beam divergence $\psi_{0}=$ $0.1 \mathrm{mrad}$. As it follows from comparison of the figure 6 and the figure 7, the multiple electron scattering in a periodic layered medium leads to a significant decrease in the amplitude of the DTR spectrum, while the width of the spectrum does not change.

Figure 8 and figure 9 show the curves constructed correspondently by formulas (3.4) and (4.3b), which describe the angular densities of the DTR for an electron rectilinearly moving along the axis $\mathbf{e}_{1}$ (figure 4) and for a divergent electron beam, considering multiple scattering. From a comparison of these figures, it follows that multiple scattering under these conditions will significantly affect the angular density of the DTR.

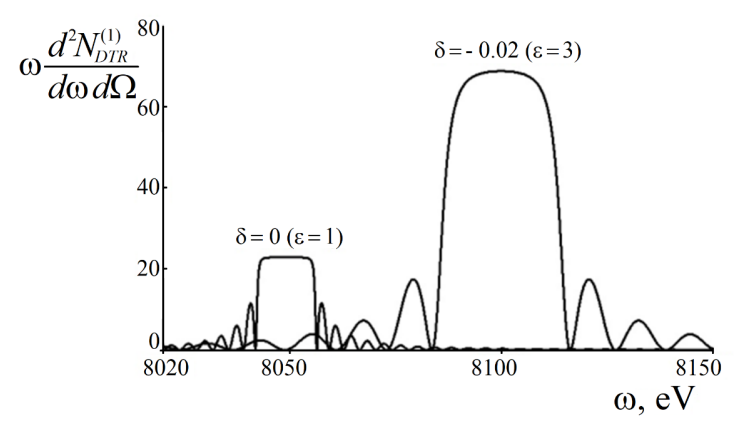

Figure 6. Spectral-angular density of DTR for cases of symmetric and asymmetric reflection for fixed observation angle $\boldsymbol{\theta}, \theta_{\perp}=\gamma^{-1}=2 \mathrm{mrad}, \theta_{\|}=0$.

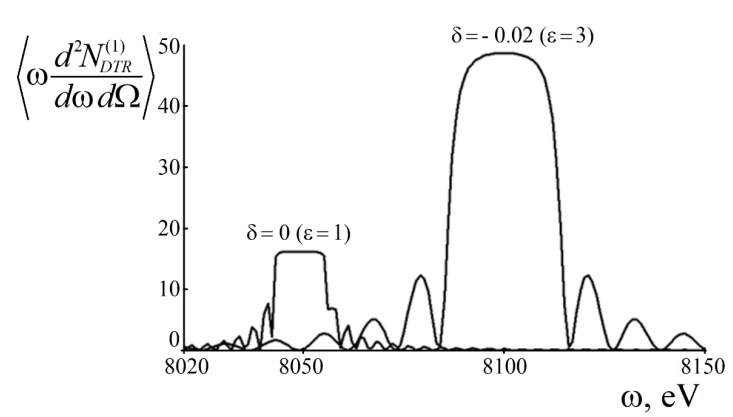

Figure 7. The same as in figure 2 but considering the beam electrons multiple scattering. The beam divergence $\psi_{0}=0.1 \mathrm{mrad}$.

Figure 8 and figure 9 show the curves constructed correspondently by formulas (3.4) and (4.3b), which describe the angular densities of the DTR for an electron rectilinearly moving along the axis $\mathbf{e}_{1}$ (figure 4) and for a divergent electron beam, considering multiple scattering. From a comparison 
of these figures, it follows that multiple scattering under these conditions will significantly affect the angular density of the DTR. But a significant effect of the asymmetry of reflection on the angular density of DTR will occur under conditions of multiple scattering as well. The effect of multiple scattering on the angular density of a DTR is a rather important effect, which is currently not experimentally registered both for periodic layered media and single crystals.

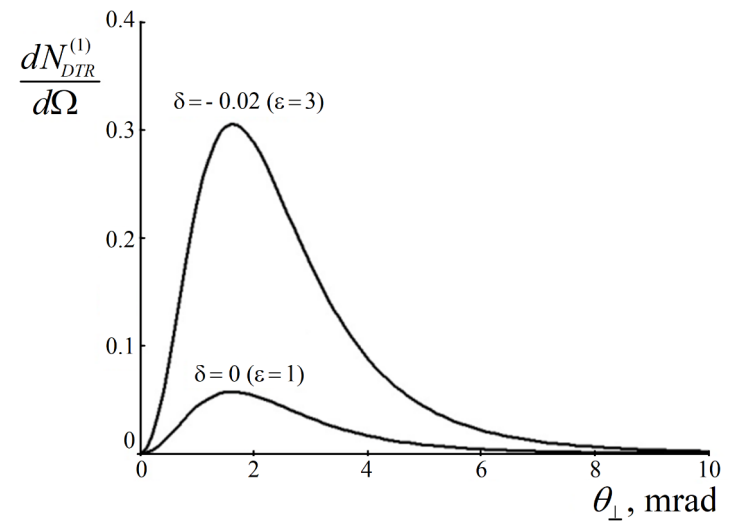

Figure 8. Angular density of PXR for symmetric $(\delta=0, \varepsilon=1)$ and asymmetric $(\delta=-0.02, \varepsilon=3)$ reflection.

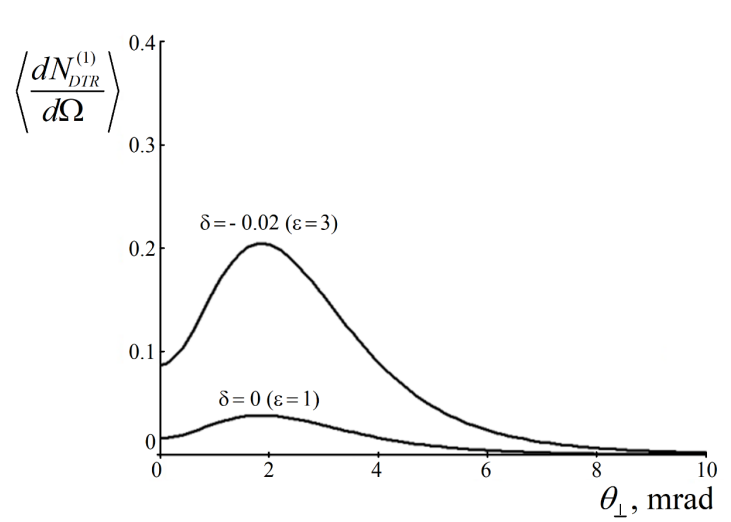

Figure 9. The same as in figure 8 with considering the effect of the electron multiple scattering.

\section{Conclusions}

We have considered the coherent X-ray radiation generated by a beam of relativistic electrons in the target with structure of a periodic layered medium in the Bragg scattering geometry for a general case of asymmetric reflection of the relativistic electron Coulomb field on the layers of the medium. We have studied the influence of the reflection asymmetry on the spectral-angular and angular characteristics of the PXR and DTR excited by beams of relativistic electrons. In particular, we show that with a decrease in the angle between the axis of the beam of relativistic electrons and the target surface, at a fixed Bragg angle, the width of the PXR spectrum increases, which leads to a significant increase in the angular density of the PXR. This effect is retained upon multiple scattering of relativistic electrons by target atoms. The multiple electron scattering in a periodic layered medium leads to a substantial increase in the width of the PXR spectrum and a decrease in its amplitude. However, under the considered conditions the electron multiple scattering affects the PXR angular density only at small viewing angles. The effect of reflection asymmetry takes place in the spectral-angular and angular densities of DTR as well. The multiple scattering substantially affects the spectral-angular and angular densities of the DTR but does not destroy the effect of reflection asymmetry. The calculations carried out for concrete parameters of target and relativistic electron beam demonstrate the influence of reflection asymmetry on the spectral-angular and angular densities of the PXR and DTR even under presence of multiple electron scattering on the atoms of the target.

The obtained results can be used as the starting data for setting an experiment on verification of theoretically predicted dynamical effects in coherent radiation excited by relativistic electrons in periodic layered media. 


\section{References}

[1] G.M. Garibian and C. Yang, Quantum microscopic theory of radiation by a charged particle moving uniformly in a crystal, Sov. Phys. JETP 34 (1972) 495.

[2] V.G. Baryshevsky and I.D. Feranchuk, Transition radiation of $\gamma$-rays in a crystal, Sov. Phys. JETP 34 (1972) 502.

[3] A. Caticha, Transition-diffracted radiation and the Cěrenkov emission of X-rays, Phys. Rev. A 40 (1989) 4322.

[4] N.N. Nasonov, Influence of the density effect upon the parametric x-rays of high energy particles, Phys. Lett. A 246 (1998) 148.

[5] N.N. Nasonov, V.V. Kaplin, S.R. Uglov, M.A. Piestrup and C.K. Gary, X rays from relativistic electrons in a multilayer structure, Phys. Rev. E 68 (2003) 036504.

[6] V.V. Kaplin et al., Observation of bright monochromatic x rays generated by relativistic electrons passing through a multilayer mirror, Appl. Phys. Lett. 76 (2000) 3647.

[7] S.V. Blazhevich, I.V. Kolosova and A.V. Noskov, Coherent $x$-ray radiation generated by a relativistic electron in an artificial periodic structure, J. Exp. Theor. Phys. 114 (2012) 547.

[8] S.V. Blazhevich and A.V. Noskov, Dynamic theory of coherent X-radiation of relativistic electron within a periodic layered medium in Bragg scattering geometry, Nucl. Instrum. Meth. B 309 (2013) 70 .

[9] S.V. Blazhevich and A.V. Noskov, Coherent X-ray radiation generated by a relativistic electron beam in a periodic layered medium in the Bragg scattering geometry, J. Exp. Theor. Phys. 125 (2017) 223.

[10] Particle Data Group, Review of particle physics. Particle Data Group, Phys. Rev. D 54 (1996) 1. 